\title{
Electronic Structure of Alkyl-Passivated Si Nanoparticles: Synchrotron-Radiation Photoemission Study
}

\author{
Tadafumi Kamikake, Masaki Imamura, Yoshiaki Murase, Akinori Tanaka, and \\ Hidehiro Yasuda \\ Department of Mechanical Engineering, Kobe University, Nada-ku, Kobe 657-8501, Japan \\ FAX: +81-78-803-6123, e-mail: a-tanaka@mech.kobe-u.ac.jp
}

We have synthesized the alkyl-passivated $\mathrm{Si}$ nanoparticles by a solution route, and have carried out the various spectroscopic studies in order to investigate their intrinsic electronic structures and surface chemistry. Photoluminescence (PL) spectra of the $n$-butyl-passivated Si nanoparticles exhibit the strong ultraviolet-blue emission. Moreover, it is found that their valence-band maximum energies directly estimated from the synchrotron-radiation valence-band photoemission spectra correspond to the resonance features in the PL excitation spectra. Therefore, it is concluded that the PL from the present Si nanoparticles originates from the electron-hole pair recombination between the modified valence-band and conduction-band due to the quantum size effect. From the synchrotron-radiation $\mathrm{Si} 2 p$ core-level photoemission spectra, their interfacial electronic structures of these Si nanoparticles have been characterized. From these results, we discuss the detailed electronic and chemical properties of alkyl-passivated Si nanoparticles.

Key words: surface-passivated Si nanoparticles, photoemission spectroscopy, electronic structure

\section{INTRODUCTION}

Semiconductor nanostructures are attracting much interest from the viewpoints of both device and fundamental physics, because they show the distinctive physical and chemical properties found in neither bulk nor molecular/atomic systems [1]. Especially, the various $\mathrm{Si}$ nanostructures have a great interest, since it has been reported that they show a strong visible photoluminescence (PL) [2] and there is a possibility of future integrating electronic devices with optical sensing technique. Numerous papers focused on the optical properties of Si-based nanostructured materials prepared by the various methods have been reported to date, however, these optical properties in the literatures depend on the samples and are suggested to ascribe to various sources such as quantum confinement effect, oxide-related species, surface state, molecular species, or impurities, etc. [3] Therefore, the interplay between the intrinsic quantum confinement effects and extrinsic surface/interface properties is still controversial. In order to understand the intrinsic properties of these Si-based nanostructured materials, it is indispensable to prepare the well-defined samples and to directly characterize their intrinsic electronic structures. In this work, we have synthesized the $n$-butyl-passivated $\mathrm{Si}$ nanoparticles with well crystalline nature and well surface-passivated surface by the solution routes [4], and have performed the various spectroscopic characterizations. Photoemission spectroscopy has been used to directly investigate the electronic structure, but to our knowledge there is no report that highlights the electronic structures of surface-passivated $\mathrm{Si}$ nanoparticles. In this paper, we have directly characterized their electronic structures and interface properties of alkyl-passivated $\mathrm{Si}$ nanoparticles by means of synchrotron-radiation based photoelectron spectroscopies. From these results, we will discuss the detailed electronic and chemical properties of these surface-passivated Si nanoparticles.

\section{EXPERIMENT}

The alkyl-passivated $\mathrm{Si}$ nanoparticles used in this work were synthesized by the oxidation of magnesium silicide $\left(\mathrm{Mg}_{2} \mathrm{Si}\right)$ with bromine and the subsequent passivation of $\mathrm{Si}_{\mathrm{m}} \mathrm{Br}$ product (Br-capped $\mathrm{Si}$ nanoparticle) with use of an alkyllithium reagent. Bromine $(1 \mathrm{ml})$ and magnesium silicide $(200 \mathrm{mg})$ were added to degassed octane $(150 \mathrm{ml})$. After stirring for 2 hours, this mixture was refluxed for 60 hours, and then the solvent and remaining $\mathrm{Br}_{2}$ were removed under reduced pressure. After that, alkyllithium $(10 \mathrm{ml})$ was added and stirred at room temperature for 2 days, and then the solvent was evaporated in a rotary evaporator. Then, an aqueous solution of hydrochloric acid was added to dissolve and remove any salts. After hexane was added, this mixture was transferred to a separatory funnel and then was washed three times with water. Finally, the hexane phase containing the Si nanoparticle was corrected, and a size-selective ultrafiltration treatment for the above crude samples was performed in order to improve the nanoparticle size distribution. The size distributions in diameter of the synthesized alkyl-passivated Si nanoparticles were characterized by ex-situ transmission electron microscope (TEM) observations. As a further characterization, Fourier transform infrared (FTIR), PL, and PL excitation (PLE) spectra were measured at room temperature. Synchrotron-radiation photoemission measurements were carried out at BL-5U of UVSOR-II Facility, Institute for Molecular Science, Okazaki, Japan. For the synchrotron-radiation photoemission measurements, the synthesized alkyl-passivated $\mathrm{Si}$ nanoparticles were supported on the highly oriented pyrolytic graphite (HOPG) substrates by evaporating the solvent from the dispersion of alkyl-passivated Si nanoparticles on the 
single-crystalline HOPG cleaved surface in a $\mathrm{N}_{2}$-filled glove bag directly connected to the ultrahigh-vacuum photoelectron spectrometer. Then the samples were transferred into the photoemission analysis chamber without exposure to air. In order to avoid the synchrotron-radiation damage, the synchrotron-radiation intensity was reduced such that the photoemission spectra showed no change in the course of the measurements.

\section{RESULTS AND DISCUSSION}

From the TEM observations (not given here), the mean diameters of $n$-butyl passivated Si nanoparticles used this work are less than $2 \mathrm{~nm}$ and less than $1 \mathrm{~nm}$. Moreover, high-resolution TEM micrographs of the present samples show the identical lattice fringes to bulk Si crystallite, indicative of well crystalline nature. In addition, FTIR spectra of the present Si nanoparticles (not given here) show no spectral features around $1000-1100 \mathrm{~cm}^{-1}$ wavenumber, attributed to $\mathrm{Si}-\mathrm{O}$ stretching modes.

Figure 1 shows the synchrotron-radiation valence-band photoemission spectra of the $n$-butyl-passivated Si nanoparticle with mean diameter less than $2 \mathrm{~nm}$ and less than $1 \mathrm{~nm}$ on the HOPG substrate at room temperature. Previously, the calculated density of states by DV-X $\alpha$ method and experimental angle-resolved photoemission spectra of $\mathrm{Si}(111)$ surface regularly terminated by methyl groups have been reported by Miyadera $e t$ al. [5]. From the analogy with these results, the spectral features in the photoemission spectrum around 7 and $5.5 \mathrm{eV}$ in binding energies originate from the $\mathrm{C} 2 p$-derived states. The spectral feature around $7 \mathrm{eV}$ in binding energy originate from the $\mathrm{C}-\mathrm{H}$ bonds in the butyl surface-passivants, and that around $5.5 \mathrm{eV}$ in binding energy originate from the $\sigma_{\mathrm{Si}-\mathrm{C}}$ bonds between the $\mathrm{Si}$ nanoparticle and butyl surface-passivants. The spectral features around $3.5,9$, and $11.5 \mathrm{eV}$ in binding energies originate from the $\mathrm{Si} 3 s$ - and $3 p$-derived states. The additional feature around $13.5 \mathrm{eV}$ in binding energy corresponds to that from an uncovered region of $\mathrm{Si}$ nanoparticles on the HOPG substrate. On the other hand, PL spectra of the present $n$-butyl-passivated $\mathrm{Si}$ nanoparticles with mean diameters less than $2 \mathrm{~nm}$ and less than $1 \mathrm{~nm}$ exhibit the distinct emissions around 3.3 and $4.0 \mathrm{eV}$ in photon energy, respectively. Moreover, the monotonic shifts of these emissions as a function of excitation photon energy have been observed (not given here). The corresponding PLE spectra of the present $n$-butyl-passivated Si nanoparticles with mean diameters less than $2 \mathrm{~nm}$ and less than $1 \mathrm{~nm}$ exhibit the distinct resonances around 4 and $4.7 \mathrm{eV}$ photon energy, respectively, and the monotonic shifts of these resonances as a function of emission photon energy have been also observed (not given here). These resonance energies in the PLE spectra of the present $\mathrm{Si}$ nanoparticles are larger than the bulk $S i$ band gap energy. From the TEM observations, it has been found that both samples have a finite size distributioni If the quantum
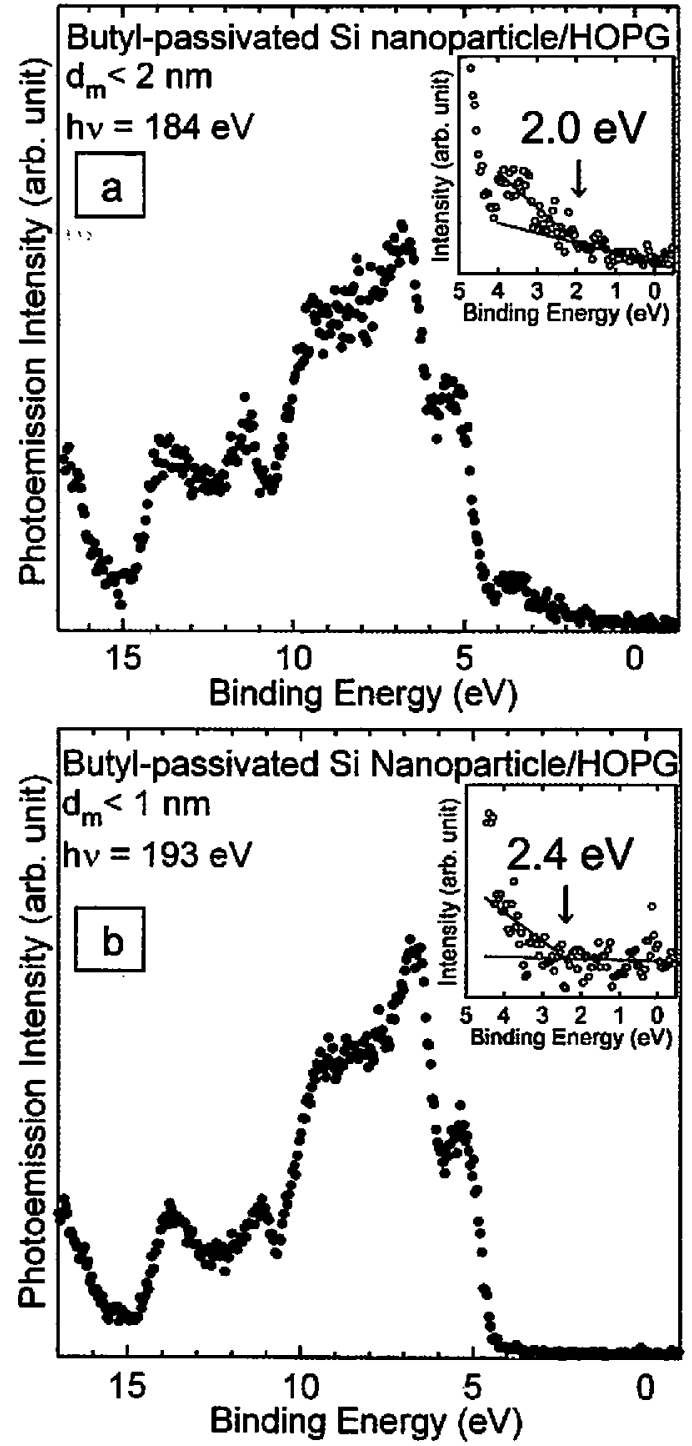

Fig. 1. The synchrotron-radiation valence-band photoemission spectra of the $n$-butyl-passivated Si nanoparticle with mean diameter less than $2 \mathrm{~nm}$ (a) and less than $1 \mathrm{~nm}$ (b) on the HOPG substrate at room temperature. Photoemission spectra in the vicinity of Fermi level of the both samples on an expanded scale are shown in the inset.

confinement effect is revealed in the both samples, the energy gap will depend on the nanoparticle size. Therefore, the monotonic shifts of PL spectra of the present samples with excitation photon energy as described above are considered to originate from the excitation of Si nanoparticles with different sizes that have different optical transition energies. That is, it is considered that the present PL observed for the $n$-butyl-passivated $\mathrm{Si}$ nanoparticles originate from electron-hole pair recombination between the modified conduction-band and valence-band due to the quantum size effect. In the inset of Fig. 1, we also plot the photoemission spectra in the vicinity of Fermi level of the both samples on an expanded scale in order to clarify 
the electronic structures in the vicinity of Fermi level. In this energy region, the spectral intensity from the HOPG substrate (uncovered region of Si nanoparticles), which reflects the vanishing semimetallic density of states toward the Fermi level, contributes to the photoemission spectra. Moreover, as shown in the inset of Fig. 1, the additional spectral intensity can be observed on the photoemission intensity from HOPG substrate. Since no contribution of $\mathrm{C} 2 s$ - and $2 p$-derived states from butyl molecular orbitals appears in this energy region, these additional intensities are considered to originate from the valence-band structures of the present $\mathrm{Si}$ nanoparticles. As shown in Fig. 1, we have estimated the valence-band maximum (VBM) energy of each sample from an intersection point of two lines extrapolated by the least-squares method of spectral tail of the valence-band structure of $\mathrm{Si}$ nanoparticles and the background signal from the HOPG substrate. From this analysis, we have obtained the VBM of about 2 and 2.4 $\mathrm{eV}$ below the Fermi level for Si nanoparticles with mean diameters less than $2 \mathrm{~nm}$ and less than $1 \mathrm{~nm}$, respectively. If each Fermi level is considered to be located in the middle of their energy gaps such as intrinsic semiconductor, the energy gaps of the present $n$-butyl-passivated Si nanoparticles with mean diameter less than $2 \mathrm{~nm}$ and less than $1 \mathrm{~nm}$ can be estimated to about 4 and $4.8 \mathrm{eV}$, respectively. These values correspond to the resonance energies in the PLE spectra fairly well.

The surface chemical-state dependence of electronic structures of hydrogen-passivated $\mathrm{Si}$ nanoparticles by means of quantum Monte Carlo calculations has been previously reported by Puzder et al. [6] They have reported that significant changes in the energy gap of fully hydrogen-passivated Si nanoparticles when the surface contains passivants other than hydrogen, in particular atomic oxygen. That is, the energy gap of fully hydrogen-passivated $\mathrm{Si}$ nanoparticles is reduced due to the multiple oxygen contamination. In addition, recently the results of $a b$ initio calculations using density functional theory for the various surface-passivated $\mathrm{Si}$ nanoparticles have been reported by Reboredo et al. [7] In this report, they have concluded small change of energy gaps of surface-passivated $S i$ nanoparticles as a function of surface passivation, in particular when the $\mathrm{Si}-\mathrm{H}$ bond (that is, hydrogen-passivated Si nanoparticle) is replaced by a $\mathrm{Si}-\mathrm{C}$ bond (that is, alkyl-passivated $\mathrm{Si}$ nanoparticle). These theoretical results for surface-passivated $\mathrm{Si}$ nanoparticles without oxygen contamination agree well with the resonance energies in the PLE spectra and photoemission spectra of both samples. Therefore, it is concluded that the present $\mathrm{PL}$ from perfectly $n$-butyl-passivated $\mathrm{Si}$ nanoparticles originates from the intrinsic quantum size effect and the modified electronic structures due to the intrinsic quantum confinement effect are directly confirmed by the present synchrotron-radiation valence-band photoemission spectra.

In order to investigate the surface-chemical properties
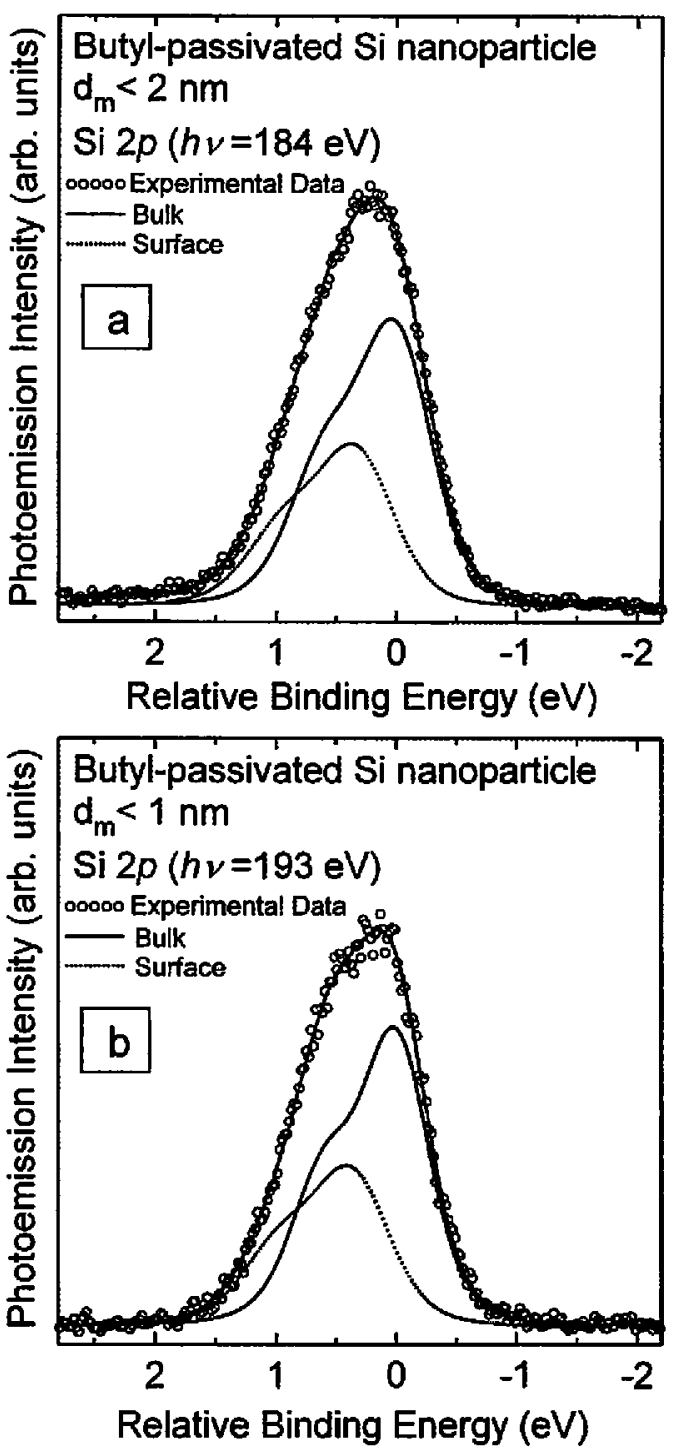

Fig. 2. The synchrotron-radiation $\mathrm{Si} 2 p$ core-level photoemission spectra of $n$-butyl-passivated $\mathrm{Si}$ nanoparticles with mean diameters less than $2 \mathrm{~nm}$ (a) and less than $1 \mathrm{~nm}$ (b). The spectra are decomposed into bulk component (solid line) and surface component bonded to surface $n$-butyl molecules (dotted line).

of the present $n$-butyl-passivated Si nanoparticles, we have observed the $\mathrm{Si} 2 p$ core-level photoemission spectra using synchrotron radiation. Figure 2 shows synchrotron-radiation $\mathrm{Si} 2 p$ core-level photoemission spectra of $n$-butyl-passivated Si nanoparticles with mean diameters less than $2 \mathrm{~nm}$ and less than $1 \mathrm{~nm}$. Figure 2 also shows the results of line-shape analyses by means of least-squares method for these experimental $S i 2 p$ core-level photoemission spectra. Each decomposed spin-orbit doublet was described by a convolution of Lorentzian line shape with a Gaussian due to the instrumental and phonon broadening. As shown in Fig. 2, it is found that the Si $2 p$ core-level spectra of the both samples are reproduced by two components fairly well. 
To date, the Si $2 p$ core-level photoemission studies on the various alkyl-terminated bulk-Si(111) surfaces have been reported $[8,9]$. From the analogy with the previous results of alkyl-terminated bulk-Si(111) surfaces, the lower-binding-energy (solid line) and higher-binding-energy (dotted line) peaks originate from the inner $\mathrm{Si}$ atoms of $\mathrm{Si}$ nanoparticles (bulk component) and surface $\mathrm{Si}$ atoms of $\mathrm{Si}$ nanoparticles bonded to surface-passivants of $n$-butyl groups (surface component), respectively. As shown in Fig. 2, these surface components accompany with the chemical shifts to higher binding energies relative to the bulk components. This indicates the different chemical states in the surface $\mathrm{Si}$ atoms bonded to $n$-butyl groups with the inner $\mathrm{Si}$ atoms and existence of a chemical reaction (chemisorption) between the surface-passivants of $n$-butyl groups and Si nanoparticles. That is, the charge transfer occurs from the core $\mathrm{Si}$ nanoparticles to surface-passivants of $n$-butyl groups due to the relative electronegativities of $\mathrm{Si}$ and $\mathrm{C}$ (1.9 and 2.55, respectively [9]). It has been reported in the previous work [8] that the chemical shifts of the methyl- $\left(\mathrm{CH}_{3}-\right)$, pentyl- $\left(\mathrm{C}_{5} \mathrm{H}_{11^{-}}\right)$, and decyl- $\left(\mathrm{C}_{10} \mathrm{H}_{2 !}-\right)$ bonded $\mathrm{Si}$ atoms of the various alkyl-terminated bulk-Si(111) surface are $0.27,0.21$, and $0.21 \mathrm{eV}$, respectively. On the other hand, the chemical shifts of the $n$-butyl-bonded $\mathrm{Si}$ atoms of the present Si nanoparticles with mean diameter less than 2 $\mathrm{nm}$ and less than $1 \mathrm{~nm}$ is $0.33 \mathrm{eV}$ and $0.36 \mathrm{eV}$, respectively. An important point to note is that the chemical shifts of the surface components in the present Si nanoparticles are significantly larger than those of surface components of alkyl-terminated bulk Si surfaces and increase with decreasing the nanoparticle diameter. Therefore, it is considered that the bonding natures between the surface-passivants of alkyl groups and surface Si atoms of Si nanoparticles are different from that of bulk surface and depend on the nanoparticle size. The number ratio of the surface Si atoms and the inner $\mathrm{Si}$ atoms which contribute to bulk components is almost same among both samples, since the present photoemission measurements are very surface sensitive. Therefore, the relative intensity ratio of surface components to bulk ones is almost same among both samples.

\section{CONCLUSION}

We have synthesized the $n$-butyl-passivated $\mathrm{Si}$ nanoparticles with crystalline nature and well-defined surface by a solution route, and have performed the synchrotron-radiation photoemission studies. From the synchrotron-radiation 'valence-band photoemission spectra of the n-butyl-passivated $\mathrm{Si}$ nanoparticles with mean diameters less than $2 \mathrm{~nm}$ and less than $1 \mathrm{~nm}$, the detailed valence-band structure of each sample is clarified, and we have estimated the VBM energy of each sample from the photoemission spectrum in the vicinity of Fermi level. It is found that these VBM energies correspond to the resonance features in the PL excitation spectra. Therefore, it is considered that the
PLs from the present $n$-butyl-passivated Si nanoparticles are originated from the electron-hole pair recombination between the modified valence-band and conduction-band due to the quantum size effect. From the synchrotron-radiation Si $2 p$ core-level photoemission spectra, it is found that the $\mathrm{Si} 2 p$ core-level photoemission spectra consists of two components, which originate from the inner $\mathrm{Si}$ atoms of $\mathrm{Si}$ nanoparticles and surface $\mathrm{Si}$ atoms bonded to surface-passivants of $n$-butyl groups. Furthermore, it is found that the chemical-shifts of these surface components are significantly larger than those of surface components of alkyl-terminated bulk-Si surfaces and increase with decreasing the nanoparticle diameter. This indicates that the bonding natures between the surface-passivants of alkyl groups and surface $\mathrm{Si}$ atoms are different among the bulk $\mathrm{Si}$ surface and $\mathrm{Si}$ nanoparticle surface and depend on the nanoparticle size.

\section{Acknowledgements}

This work was supported by Grant-in-Aids from the Ministry of Education, Culture, Sports, Science and Technology of Japan. This work was also supported by the grants from Hyogo Science and Technology Association and Kawanishi Memorial Shinmaywa Education Foundation. Synchrotron-radiation experiment was performed under the Joint Studies Program (2004, 2005, and 2006) of the UVSOR-II Facility, Institute for Molecular Science. We thank T. Ito, S. Kimura, and the staffs of UVSOR-II Facility, Institute for Molecular Science, for their technical support. We also thank S. M. Kauzlarich and K. A. Pettigrew of Department of Chemistry, University of Califomia, Davis, for useful discussions.

\section{REFERENCES}

[1] For example, A. Tanaka, S. Onari, and T. Arai, Phys. Rev. B, 47, 1237-1243 (1993).

[2] For example, L. T. Canham, Appl. Phys. Lett., 57 1046-1048 (1990).

[3] For example, A. G. Cullis, L. T. Canham, and P. D. Calcott, J. Appl. Phys, 82, 909-965 (1997).

[4] K. A. Pettigrew, Q. Liu, P. P. Power, and S. M. Kauzlarich, Chem. Mater., 15, 4005-4011 (2003).

[5] T. Miyadera, A. Koma, and T. Shimada, Surf. Sci., 526, 177-183 (2003).

[6] A. Puzder, A. J. Williamson, J. C. Grossman, and Griulia, Phys. Rev. Lett., 88, 097401-1-097401-4 (2002). [7] F. A Reboredo and G. Galli, J. Phys. Chem. B, 109, 1072-1078 (2005).

[8] J. Terry, M. R. Linford, C. Wigren, R. Cao, P. Pianetta, and C. E. D. Chidsey, J. Appl. Phys., 85, 213-221 (1999).

[9] R. Hunger, R. Fritsche, B. Jaeckel, W. Jaegermann, L. J. Webb, and N. S. Lewis, Phys. Rev. B, 72, 045317 $-1-045317-7(2005)$.

(Received December 10, 2006; Accepted March 8, 2007) 